\title{
Controlled chemistry of tailored graphene nanoribbons for electrochemistry: a rational approach to optimizing molecule detection
}

\author{
Aida Martín ${ }^{a}$, Javier Hernández-Ferrer ${ }^{b}$, Luis $\operatorname{Vázquez}^{c}$, María Teresa Martínez $^{b}$ and Alberto Escarpa ${ }^{* a}$ \\ Received (in $X X X, X X X)$ Xth $X X X X X X X X X 20 X X$, Accepted Xth XXXXXXXXX 20XX \\ ${ }_{5}$ DOI: 10.1039/b000000x
}

This work describes a rationalization of the interactions between two fully characterized graphene nanoribbons (GNRs) and a set of significant target molecules. The GNRs were carefully synthesized by unzipping multi-walled carbon nanotubes (MWCNTs) to yield graphene oxide nanoribbons (GNRox) containing $44 \mathrm{wt} . \%$ oxygen. The GNRox were reduced to yield reduced graphene oxide nanoribbons

10 (GNRred) containing $14 \mathrm{wt} . \%$. Each material was characterized by atomic force microscopy, transmission electronic microscopy, Raman spectroscopy, X-ray diffraction, Fourier transform infrared spectroscopy, $\mathrm{X}$-ray photoelectron spectroscopy and voltammetry techniques. Differential pulse voltammetry was used to assess the detection of two strategically selected groups of molecules, including benzenediols, hydroquinone, catechol, and resorcinol, as well as, L-dopa, ascorbic acid, uric acid, and L-tyrosine. The 15 results showed that GNRs provided significantly better electrochemical responses compared to MWCNTs and the non-modified glassy carbon electrode. The chemistry of the few layers of graphene strongly influenced the electrochemical properties of the material. GNRox may be the material of choice for sensing molecules having high oxidation potentials. GNRred, on the other hand, yielded an excellent sensitivity for aromatic molecules in which $\pi-\pi$ interactions were dominant or the number of conjugated 20 1,2-diols present was high. GNRred combines the advantages of the high proportion of $\mathrm{sp}^{2}$-carbon atoms with the presence of a few oxygen moieties remaining in the lattice after the reduction step. The primary interactions responsible for the shift in oxidation potentials were elucidated. This work presents new opportunities for tailoring graphene to a particular sensing application based on the specific chemistry of the molecule.

\section{${ }_{25}$ INTRODUCTION}

Graphene is a two-dimensional (2-D) sheet of carbon atoms connected by $\mathrm{sp}^{2}$ bonds. The graphene structure conveys extraordinary properties ${ }^{1-3}$ to the material, such as a high surface area (theoretically $2630 \mathrm{~m}^{2} / \mathrm{g}$ for single-layer graphene) that is 30 twice the surface area of single-walled carbon nanotubes (SWCNTs). Graphene also shows excellent thermal $\left(\mathrm{k}=5 \times 10^{3}\right.$ $\left.\mathrm{Wm}^{-1} \mathrm{~K}^{-1}\right)$ and electrical conductivities $\left(\sigma=64 \mathrm{mS} \mathrm{cm}^{-1}\right)$. The physical properties of graphene include good optical transparency, a high mechanical strength (Young's modulus, $35 \sim 1100 \mathrm{GPa}$ ), and a high elasticity. The high surface area, high electrical conductivity, and low production costs, in particular, are of interest for electrochemical applications.

Graphenes include graphitic structures that are dimensionally limited to a few to hundred nanometres along the basal plane of 40 the graphene sheets in the $\mathrm{x}-\mathrm{y}$ plane, such as graphene nanoribbons (GNRs), which can including single- (G-SL), few(G-FL), or multilayer (G-ML) structures ${ }^{4}$. GNRs may be thought of as unzipped carbon nanotubes (CNTs) that have been created with structural control during the unzipping process. The ${ }_{45}$ synthesis of GNR ${ }^{5,6}$ is carried out by applying plasma etching to
CNTs embedded in a polymer film ${ }^{7}$, by chemically oxidizing CNTs ${ }^{8,9}$, by ionic liquid-assisted splitting of CNTs under microwave radiation ${ }^{10}$, by the intercalation of metals ${ }^{11}$ or nanoparticles ${ }^{12}$ into CNTs, and the posterior exfoliation or 50 unwrapping of MWCNTs using electrical currents and nanomanipulation ${ }^{13}$, or by bottom-up strategies ${ }^{14}$ for producing GNRs of a desired width and length. The main applications ${ }^{15}$ of GNRs occur in the fields of physics, nanoelectronics, spintronics, and nanoelectromechanical systems (NEMS) ${ }^{16}$, but GNRs are 55 also relevant to sensing and biosensing applications.

Although the longitudinal unzipping techniques used to synthesize GNRs result in over-oxidation and a plethora of defect sites that do not in general benefit electronic applications ${ }^{17}$, these characteristics can actually be useful for certain electrochemical 60 applications, as the authors have proved.

Electrochemical applications using graphene have been extensively discussed in different reviews reported in the literature ${ }^{18-21}$.

On the other hand, the corresponding applications of GNRs ${ }_{65}$ have not been extensively explored. The edge chemistries of chemically functionalized GNRs may offer certain advantages over the edge chemistries of non-functionalized graphene. Nonfunctionalized graphene presents an inert chemical surface. By 
contrast, the functional moieties located at the edges of GNRs facilitate the adsorption of molecules by $\pi-\pi$ stacking, electrostatic, hydrogen bonding, and covalent interactions ${ }^{22}$.

A handful of studies have examined the use of GNRs in 5 electrochemical sensing applications. The electrochemical sensing of model electroactive molecules in the presence of reduced GNR-modified screen-printed electrodes has been shown to display a higher sensitivity compared to the sensitivity of a bare screen-printed electrode ${ }^{17}$. Various GNR-based sensors and 10 biosensors ${ }^{23-25}$ have been developed for the detection of urea ${ }^{26}$, glucose ${ }^{27}$, 1-hydroxypyrene ${ }^{28}$, cysteine ${ }^{29}$, brevetoxin $\mathrm{B}^{30}$, and 2,4,6-trinitrotoluene ${ }^{31,32}$. These sensors displayed excellent electrochemical responses in terms of reproducibility, a low detection limit, and a high selectivity in all cases. In other 15 approaches, GNRs have been incompletely unzipped to develop mixtures of GNR/MWCNTs. The utility of these mixtures for sensing electroactive molecules ${ }^{33}$ has been examined, yielding good responses.

To explore these features mentioned above and elucidate the 20 chemical interactions between target molecules and graphene in the context of electrochemical sensing, a set of analytically significant target molecules were evaluated using differential pulse voltammetry (DPV). We tested the detection of several target molecules, including three dihydroxybenzene isomers 25 widely used in the chemical industries ${ }^{34}$ : catechol (CT), resorcinol (RS), and hydroquinone (HQ); the neurotransmitter Ldopa (Levodopa/L-3,4 dihydroxyphenylalanine, LD); the amino acid L-tyrosine (L-Tyr); uric acid (UA) and ascorbic acid (AA), which are present in urine and blood serum ${ }^{35-37}$. These target 30 molecules are usually oxidized at approximately the same potential; therefore, discrimination among these species in a mixture can be extremely difficult using most solid electrodes ${ }^{38}$.

In the bibliography interactions between molecules and graphene have been reported using density functional theory and 35 quantum physics 39,40 . The studies described here sought to (1) explore the electrochemical performances of fully characterized GNRs with respect to the detection of significant target molecules, and (2) elucidate the chemical interactions between graphenes and the target molecules. Since GNRox were obtained 40 from MWCNTs and the GNRred were obtained from the chemical reduction of GNRox, a reliable and valuable comparison with the critical controls such as the GCE and MWCNTs could be made. Therefore, this traceability is of paramount importance for ascertaining the advantages of GNRs.

\section{${ }_{45}$ MATERIALS AND METHODS}

\section{Reagents, standards and samples}

The sodium dihydrogen phosphate and disodium hydrogen phosphate used to prepare a phosphate buffered solution (PBS) and the potassium hexacyanoferrate (III) were purchased from 50 Panreac, (Badalona, Spain). LD, UA, RS, HQ, and CT were purchased from Sigma-Aldrich (St. Louis, MO, USA). AA and LTyr were obtained from Fluka Chemika (Buchs, UK). The cosmetic sample (Pigmentasa formulation containing 4\% (w/w) HQ) was acquired in a local market. Urine samples were 55 recollected from healthy patients.

Standard solutions were prepared in $1 \mathrm{mM}$ in $0.1 \mathrm{M}$ phosphate buffered solution (PBS) at $\mathrm{pH}=7.4$, adjusted using $\mathrm{NaOH}$. All working solutions were protected from light and prepared daily. A $0.1 \mathrm{~g}( \pm 0.0001)$ sample of the Pigmentasa formulation was ${ }_{60}$ diluted in $10 \mathrm{~mL}$ PBS, sonicated in an ultrasonication bath for 10 min, filtered through a $0.2 \mu \mathrm{m}$ Nylon filter, and diluted 4-fold prior to analysis. Urine samples were diluted 25 -fold prior to analysis.

All solutions were prepared with Milli-Q water produced in a ${ }_{65}$ Milli-Q system (Millipore, Bedford, MA, USA).

\section{Graphene nanoribbon samples}

MWCNTs $(0.2 \%$ oxygen content $)$ were produced by the arcdischarge method ${ }^{41}$ in a home-built electric arc-discharge apparatus under standard conditions ${ }^{42}$. The MWCNTs were 70 characterized as being straight and highly graphitized.

GNRox, 44 wt.\% oxygen, were synthesized from the MWCNTs via the longitudinal unzipping method in $\mathrm{H}_{2} \mathrm{SO}_{4} / \mathrm{KMnO}_{4}{ }^{8}$. These oxidized nanoribbons were used as starting materials to produce GNRred, $14 \%$ oxygen, via chemical 75 reduction with $\mathrm{N}_{2} \mathrm{H}_{4} / \mathrm{NH}_{3}{ }^{43}$.

\section{Apparatus and measurements}

Atomic force microscopy (AFM) images were recorded using a Nanoscope IIIa scanning probe microscope (Digital Instruments, USA) operated in the dynamic and contact modes. In both cases, 80 silicon cantilevers (Vecco) with a force constant of $\sim 40 \mathrm{~N} / \mathrm{m}$ and a nominal radius of $8 \mathrm{~nm}$ were employed. The samples used for the measurements were prepared by drop-casting $0.5 \mu \mathrm{L}$ of the graphene suspension $(0.1 \mathrm{mg} / \mathrm{mL})$ on the surface of a silicon wafer (in the case of the GNRox studies) or on a mica surface (in 85 the case of the GNRred studies). The measurements were obtained in the surroundings of the wet drop where the concentration and aggregation of graphene sheets was lower. Transmission electron microscopy (TEM) images were collected on a JEOL microscope model 2000 FXII at an acceleration 90 potential of $200 \mathrm{kV}$, which yielded a maximum resolution of 0.28 $\mathrm{nm}$. Raman spectra were obtained on a Micro-Raman confocal spectrophotometer model Horiba Jobin Yvon HR800 UV using a green laser at $532 \mathrm{~nm}$, which yielded a resolution of $0.4 \mathrm{~cm}^{-1}$. Xray photoelectron spectroscopy (XPS) was carried out on an ${ }_{95}$ ESCAPlus Omicron outfitted with a $\mathrm{Mg}$ anode and operated at $1253.6 \mathrm{eV}$ with a power of $150 \mathrm{~W}(14 \mathrm{~mA}, 10 \mathrm{kV})$. X-ray diffraction (XRD) measurements were obtained using an X-ray diffractometer Bruker D8 Advance Series. The oxygen content of the graphene samples was direct determined using a Flash 1112 100 analyzer from Thermo Fisher Scientific.

All electrochemical measurements were performed on an electrochemical station $\mu$-AUTOLAB type II (Ecochemie, Utrecht, Holland) using a conventional three-electrode system comprising a platinum wire as an auxiliary electrode, a 105 silver/silver chloride, $3 \mathrm{M} \mathrm{KCl}(\mathrm{Ag} / \mathrm{AgCl})$ reference electrode (CH Instrument, China), and a glassy carbon electrode (GCE) 3.0 $\mathrm{mm}$ in diameter (BAS Instrumental, Warwickshire, UK) as the working electrode. Electrochemical experiments were performed at room temperature.

110

\section{Procedures}




\section{Preparation of the graphene-modified electrode}

GNRox was dispersed in water by bath ultrasonication to form a $0.5 \mathrm{mg} \mathrm{mL} \mathrm{m}^{-1}$ colloidal dispersion. GNRred was dispersed to obtain a $0.5 \mathrm{mg} \mathrm{mL} \mathrm{m}^{-1}$ dispersion in water/ $\mathrm{NH}_{3}(1 \% \mathrm{v} / \mathrm{v})$ by 5 ultrasonication in a bath for $30 \mathrm{~min}$, followed by tip sonication using a VCX130, (Sonics, Newtown, USA) for 2 minutes at 130 W. Interestingly, this last step was highly important for producing excellent dispersions of the GNRred (see Fig. S1). The MWCNTs were dispersed in dimethylformamide (DMF) by 10 ultrasonication to form a $0.5 \mathrm{mg} \mathrm{mL}^{-1}$ dispersion.

Prior to drop-casting deposition, the GCE was in turn polished using 0.1 and $0.05 \mu \mathrm{M}$ alumina powders and sequentially sonicated in Milli-Q water and anhydrous ethanol. The GNR and MWCNT-modified electrodes were prepared by casting $10 \mu \mathrm{L}$ of 15 the GNR solutions (oxide or reduced) or MWCNTs dispersions on the GCE surface (see Table S1).

\section{Electrochemical procedures}

The electrochemical effective surface areas of the bare and modified GCE were estimated based on the slope of the plot of Q 20 vs. $\mathrm{t}^{1 / 2}$ obtained by chronocoulometry based on Equation 1, as described by Anson ${ }^{44,45}$, using $0.45 \mathrm{mM} \mathrm{K}_{3}\left[\mathrm{Fe}(\mathrm{CN})_{6}\right]$ in $0.1 \mathrm{M}$ PBS (pH=7.4).

$$
Q(t)=\frac{2 n F A c D^{1 / 2} t^{1 / 2}}{\pi^{1 / 2}}+Q_{d l}+Q_{a d s}
$$

(Equation 1)

In this equation, $\mathrm{A}$ is the effective electrochemical surface area 25 of the working electrode $\left(\mathrm{cm}^{2}\right), \mathrm{c}$ is the concentration of the electroactive species $\left(\mathrm{mol} / \mathrm{cm}^{3}\right), \mathrm{n}$ is the number of transfer electrons (that is, 1), F is the Faraday constant, and D is the diffusion coefficient, $7.6 \times 10^{-6} \mathrm{~cm}^{2} \mathrm{~s}^{-1}{ }^{46}$. $\mathrm{Q}_{\mathrm{dl}}$ is the double layer charge, which could be eliminated by background subtraction, 30 and $\mathrm{Q}_{\mathrm{ads}}$ is the Faradaic charge.

DPV was used for the voltammetry analysis with a pulse width of $0.05 \mathrm{~V}$, a pulse frequency of $0.05 \mathrm{~s}$, a pulse cycle of $0.2 \mathrm{~s}$, a pulse interval of $0.004 \mathrm{~V}$, and a standing time of $2 \mathrm{~s}$.

\section{RESULTS AND DISCUSSION}

\section{${ }_{35}$ Characterization of the graphene nanoribbons}

The structures and morphologies of the graphene samples were characterized using AFM, as shown in Fig. 1. This figure shows AFM images for a small GNRox sheet with an average thickness of about $0.8 \mathrm{~nm}$ and an area of $200 \times 150 \mathrm{~nm}^{2}$. The theoretical 40 thickness of a perfectly flat unoxidized $\mathrm{sp}^{2}$-carbon-atom network is predicted to be $0.4 \mathrm{~nm}{ }^{1}$. The thickness of the GNRox sample measured here was consistent the thickness of two stacked graphene sheets; however, this thickness may also indicate a single layer graphene structure having oxygen functionalities on 45 the surface ${ }^{47}$. Fig. 1 shows that the GNRred surface was rough due to the presence of stacked small fragments of the reduced graphene sheets. The average thickness of this graphene sample was $1.2 \mathrm{~nm}$, suggesting that the stacked layers extended over an area of $120 \times 50 \mathrm{~nm}^{2}$. The GNRred sample displayed a larger 50 number of layers than the graphene oxide sample because the high proportion of $\mathrm{sp}^{2}$-carbons in GNRred increased the extent of $\pi-\pi$ stacking interactions.
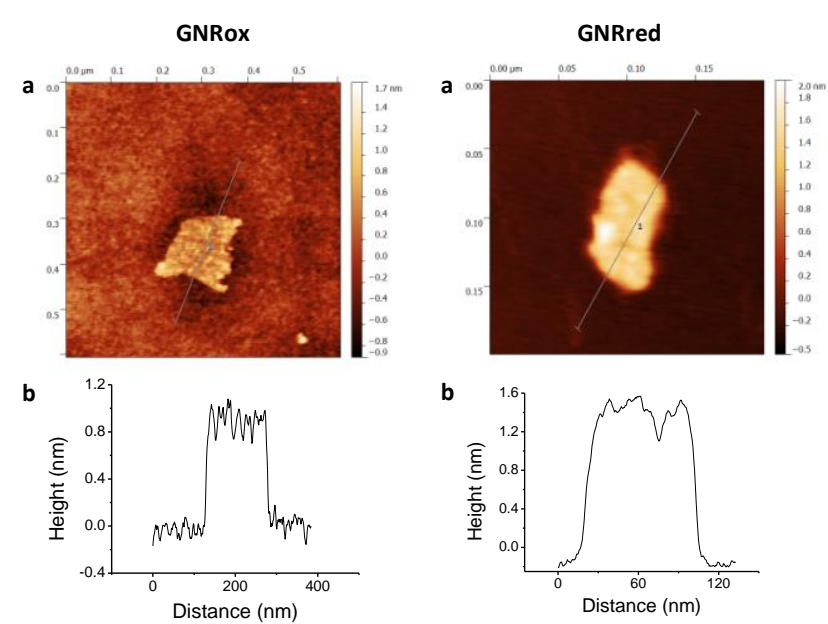

Fig. 1. (a) Tapping mode AFM images of GNRox (left) and GNRred 55 (right) on silica and freshly cleaved mica substrates, respectively. (b) Height profiles along the dashed lines indicated in the panels (a).

The graphene morphologies were imaged using TEM, which also demonstrated the successful synthetic process based on the chemical unzipping of MWCNTs. Fig. 2 shows TEM images of a 60 MWCNT sample (A), GNRox (B), and GNRred (C). Although the MWCNTs were more than $1 \mu \mathrm{m}$ long and had an outer diameter of approximately $12 \mathrm{~nm}$; GNRox displayed stacked layers and folds, and GNRred appeared to form thin layers with folds in the sheets. These micrographs could be used to visualize 65 the openings of the MWCNTs used to generate the graphene layers, as well as the anisotropy of the GNR structures.

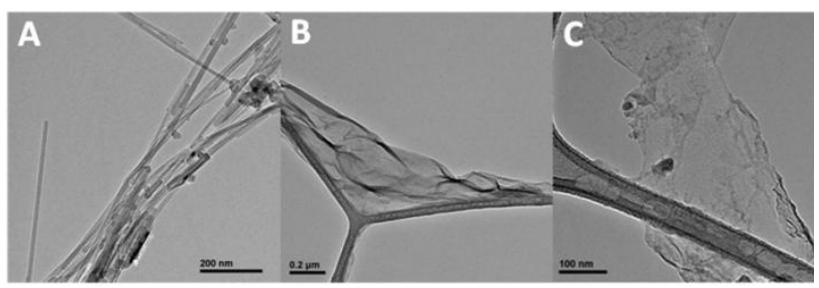

Fig. 2. TEM images of the MWCNTs (A), GNRox (B), and GNRred (C) samples.

X-Ray diffraction studies were performed to estimate the 70 average distance between layers in the carbon allotropes. The crystalline structures of graphite, graphene, and carbon nanotubes permit the measurement of the inter-planar spacing and lattice parameters (see SI, Fig. S2). The XRD patters obtained from the MWCNTs presented a peak at $26^{\circ}$ corresponding to a basal plane 75 of $\mathrm{d}_{002}=3.34 \AA$. This peak matched the distance found in the graphite layer structure. The GNRox sample displayed a new peak at $10^{\circ}$, which was attributed to a plane at $\mathrm{d}_{001}=7.33 \AA$. The separation between layers in the GNRox sample was high due to the presence of oxygen moieties in the lattice. The GNRred peak 80 intensity at $10^{\circ}$ was lower and the peak at $26^{\circ}$ was higher than the corresponding peak intensities obtained from the GNRox diffractogram. The recovery of $\mathrm{sp}^{2}$-carbon in GNRred facilitated $\pi-\pi$ stacking among the layers, and the distance between layers was smaller than the interlayer distance measured in the GNRox 85 sample. XRD studies confirmed the change in the distance between these crystalline structures and the presence of a new graphitic structure.

The Raman spectra of graphite-derived materials usually display a $\mathrm{D}$ band at $1360 \mathrm{~cm}^{-1}$ and a G band at $1590 \mathrm{~cm}^{-1}$, and an 
overtone of the $\mathrm{D}$ band occurs at $2650 \mathrm{~cm}^{-1}$ (the $2 \mathrm{D}$ or $\mathrm{G}^{\prime}$ band)

7,48 . The $\mathrm{D}$ band arises from the out-of-plane vibrational modes and is indicative of the number of $\mathrm{sp}^{3}$ carbon atoms present, whereas the $\mathrm{G}$ band arises from the presence of in-plane $\mathrm{sp}^{2}$ ${ }_{5}$ vibrations. The intensity ratio of the $\mathrm{D}$ and $\mathrm{G}$ lines $\left(\mathrm{I}_{\mathrm{D}} / \mathrm{I}_{\mathrm{G}}\right.$ ratio), therefore, provides important information about the composition and domains in-plane giving valuable information regarding the average size of the $\mathrm{sp}^{2}$ carbon domains as well ${ }^{49,50}$. Fig. S3 illustrates the Raman spectra of the three materials, showing the ${ }_{10} \mathrm{D}, \mathrm{G}$, and $\mathrm{G}$ ' bands. The $\mathrm{I}_{\mathrm{D}} / \mathrm{I}_{\mathrm{G}}$ ratio was calculated to be 0.075 , 0.52, and 0.66 for the MWCNTs, GNRox, and GNRred samples, respectively. This increase also suggested a decrease in the average size of the $\mathrm{sp}^{2}$ graphitic domains, suggesting that the new graphitic domains created in the GNRred sample were smaller in 15 size but more numerous than in the GNRox sample ${ }^{48}$. Because the $\mathrm{I}_{\mathrm{D}} / \mathrm{I}_{\mathrm{G}}$ ratio is proportional to the average size of the $\mathrm{sp}^{2}$ carbon domains, a higher $\mathrm{I}_{\mathrm{D}} / \mathrm{I}_{\mathrm{G}}$ was attributed to the presence of additional edges (more defects) ${ }^{51}$ and shorter layers in the GNRred surface, consistent with the AFM results.

20 XPS was used to study the oxygen content and changes in the $\mathrm{sp}^{2}-\mathrm{sp}^{3}$ carbon structure in the graphene layers after chemical reduction of GNRox to obtain GNRred. The presence of $\mathrm{sp}^{2}$ carbon atoms increased significantly and the presence of $\mathrm{sp}^{3}$ carbon and oxygen moieties decreased correspondingly upon 25 reduction of GNRox to GNRred. The presence of carboxyl, carbonyl, alcohol, epoxy, and ether moieties decreased to the same extent. The XPS results revealed the presence of $\mathrm{C}-\mathrm{N}$ bonds in the as-synthesized GNRred sample as a result of the $\mathrm{N}_{2} \mathrm{H}_{4} / \mathrm{NH}_{3}$ reduction step (see Fig. S4). Considering that XPS measurements 30 are sensitive to the elemental composition of the material surface, XPS offers an accurate measure of the oxygen content in the material. Direct determination of oxygen content was directly determined to be $0.2 \mathrm{wt} \%$ in the MWCNTs, $44 \mathrm{wt} \%$ in the GNRox, and 14 wt.\% in the GNRred. Clearly, these data differed 35 slightly from the XPS results because the XPS technique is not sensitive to the presence of oxygen groups below the surface and because some of the groups may be lost due to decomposition in the presence of the harsh XPS measurement conditions (X-ray irradiation under high-pressure conditions). The IR spectra were ${ }_{40}$ evaluated to corroborate the previous data (see Fig. S5).

The dispersion of the graphene material was of paramount importance for obtaining these results (see Fig. S1). Stronger sonication conditions were required to prepare the GNRred dispersion. Because GNRred includes high $\mathrm{sp}^{2}$ content, the 45 material readily stacks to form small piles on an electrode surface that increase the resistivity and reduce the current. The strongest sonication conditions were not necessary to obtain a good GNRox dispersion because the layers did not stack as readily, the material was not found to accumulate, and the signals obtained 50 with or without tip sonication were indistinguishable.

In the last analytical characterization step, the casting electrode was prepared using a $10 \mu \mathrm{L}$ drop of the GNR suspensions (see Table S1) and the effective electrochemical surface area was evaluated by chronocoulometry (see Fig. S6). The estimated 55 areas were $0.030,0.071,0.113$, and $0.267 \mathrm{~cm}^{2}$ for the nonmodified GCE, MWCNTs, GNRox, and GNRred electrodes, respectively. These results revealed that GNRox and GNRred significantly increased the electrochemical surface area of the electrode. This increase corresponded to a 4-fold increase, in the 60 case of GNRox, and a 10-fold increase, in the case of GNRred, over the electrochemical surface area of the bare GCE. The surface areas were higher than the surface area of the MWCNTs by factors of 2 and 5 for the GNRox and GNRred samples, respectively. Good interelectrode precision ( $n=3$ electrodes) was 65 obtained, with relative standard deviation (RSD) values of 2,7 , and $6 \%$ for the MWCNTs, GNRox and GNRred, respectively. These results indicated that the GNRs offered consistently high and reproducible electroactive areas, as predicted.

Interactions between the target molecules and the GNRs 70 based on voltammetry studies

The electrochemical behaviors of the isomers HQ (1,4-diol), CT (1,2-diol), and RS (1,3-diol) were explored on bare GCE, and all the carbon materials (MWCNTs, GNRox, and GNRred) on the GCE, respectively. Fig. 3 Top illustrates the electrochemical 75 responses of each electrode to the target molecules. The extraordinarily high oxidation peak currents on the graphenemodified electrodes, as compared to the bare electrode, in the presence of the three target molecules reflected the high conductivity of the GNRs. The high conductivity of the GNRred 80 electrode was particularly remarkable as a result of the high $\mathrm{sp}^{2}$ carbon content, which increased the electrical conductivity of this material. As an example, the electrochemical response of GNRred to catechol was one order of magnitude higher than the response to HQ, (see Table S2). The presence of incompletely 85 unzipped MWCNTs, if any, was not expected to significantly affect the detection performance because the MWCNT electrode displayed a low signal level. 
a

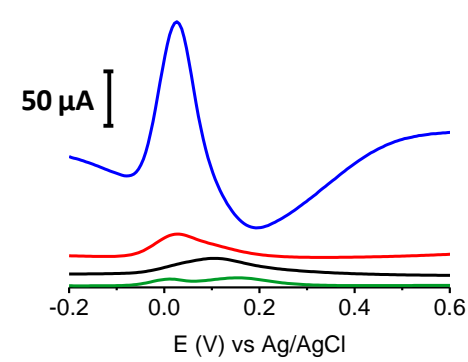

b

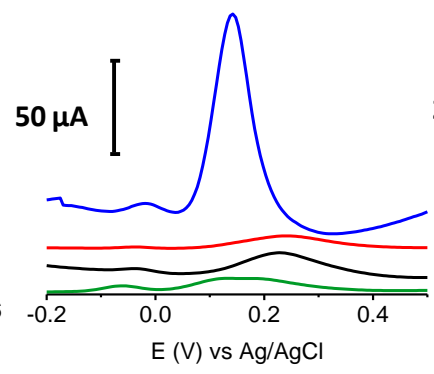

C

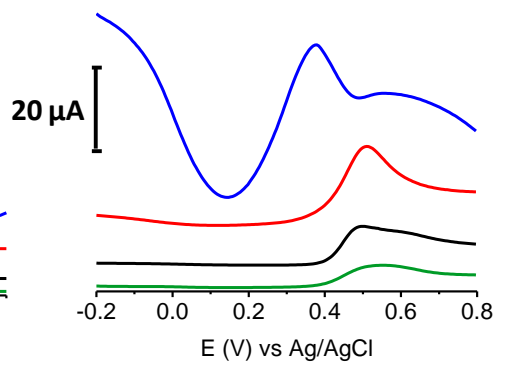

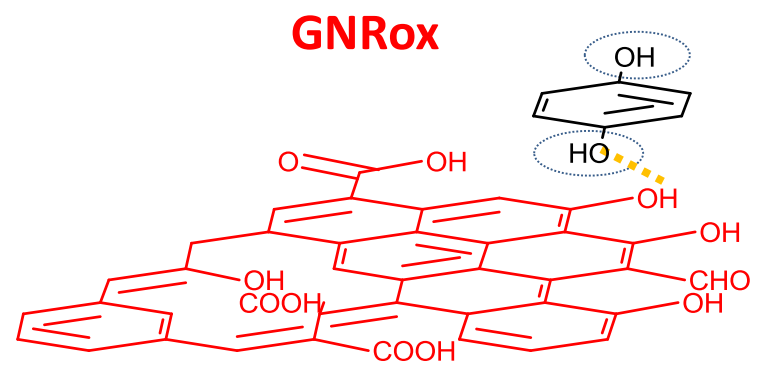

GNRred

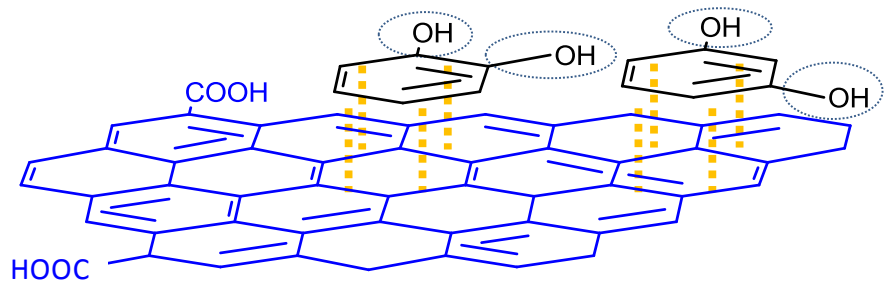

Fig. 3 (Top) Differential pulse voltammograms obtained from $1 \mathrm{mM} \mathrm{HQ}$ (a), CT (b), and RS (c). (一) Bare GCE, (一) MWCNTs, (一) GNRox, and (一) GNRred. (See the experimental section for a description of the working conditions). (Bottom) Schematic diagram of the interactions between the target molecules (black) and the graphene materials which showed the lower oxidation potential. The oxidation reaction center is indicated by the blue circles 5 and the main interactions are indicated in yellow.

The chemistry underlying the shifts in oxidation poten-tials in the electrochemical detection of the target mole-cules may be understood as follows. 1,4-Benzenediol readily oxidizes at low potentials to produce 1,4-benzoquinones, whereas the oxidation 10 of 1,3-diol is less favorable due to the lack of conjugation, thereby preventing the formation of the benzoquinone, as it occurs with 1,4 and 1,2-benzenediols. Fig. 3 Bottom illustrates the possible interaction processes between the target molecules and the graphene material. We hypothesized that the availability 15 of more $\pi-\pi$ interactions in GNRred than in GNRox facilitated the electrocatalysis of CA and RS and, as a consequence, facilitated oxidation of both benzenediols with GNRred. By contrast, 1,4benzenediol displayed similar oxidation potentials on both GNRs, suggesting that hydrogen bonds dominated the interactions 20 between the oxide moieties on the edges of the GNR surfaces and the molecules, due to the similar potential of the HQ in the presence of GNRred and GNRox.

Fig. 4 Top presents the detection results of LD, AA, L-Tyr, and UA on GCE, MWCNTs, GNRox, and GNRred. A 25 comparison of the graphene modified electrodes and the GCE and MWCNTs controls revealed that both graphene electrodes displayed significantly higher current peaks compared to the MWCNT control. The results obtained from GNRred were particularly spectacular. The exceptional electrocatalytic 30 properties exhibited by this material enabled the detection of AA, even below 0 V. Moreover, GNRred displayed analytical response from 5 to 10 -fold higher compared to the response of the GCE.

Table S3 summarizes the oxidation potentials and analytical ${ }_{35}$ signals obtained during the DPV measurements for the four molecules.

The enhanced sensitivity of the GNRred electrode was attributed to the good conductivity of the material. These results may be understood in terms of the previous results obtained in 40 analytical characterization studies, which identified a high electrochemical surface area and the presence of high $\mathrm{sp}^{2}$-carbon content. Fig. 4 Bottom provides a schematic diagram illustrating our rationalization of the interactions between the GNR and the target molecules. The oxygen groups of the GNRox layers and 45 those oxygen functionalities remaining in the GNRred lattice (this latter material supposed to be in the form of carboxylic acids and carbonyls, as described in the model proposed by Lerf-Klinowski $43,52,53$ ) are expected to interact with the target molecules via hydrogen bonds. In the detection of AA, GNRred yielded the 50 strongest electrocatalysis compared to UA, where similar electrocatalytic effect was found for both graphenes. This shift could be explained considering interactions with the oxidation center of AA and GNRred and without this oxidation center in the case of UA. 
a

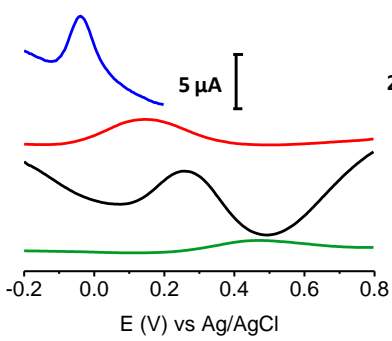

b

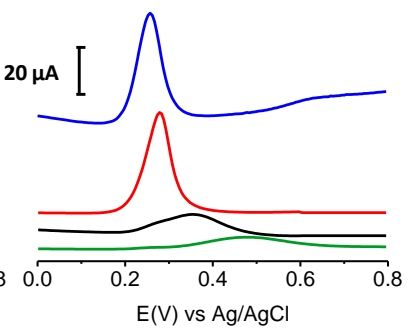

C

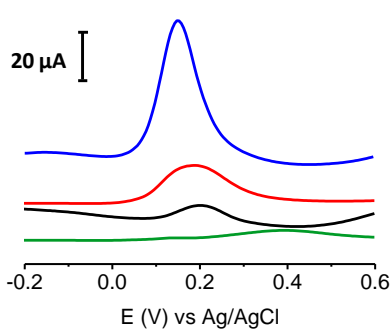

d

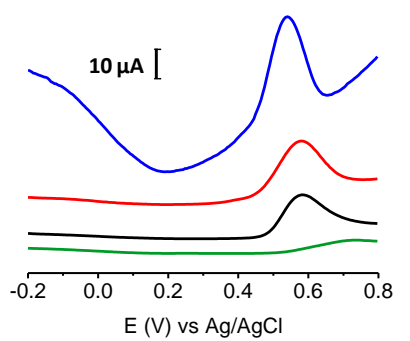

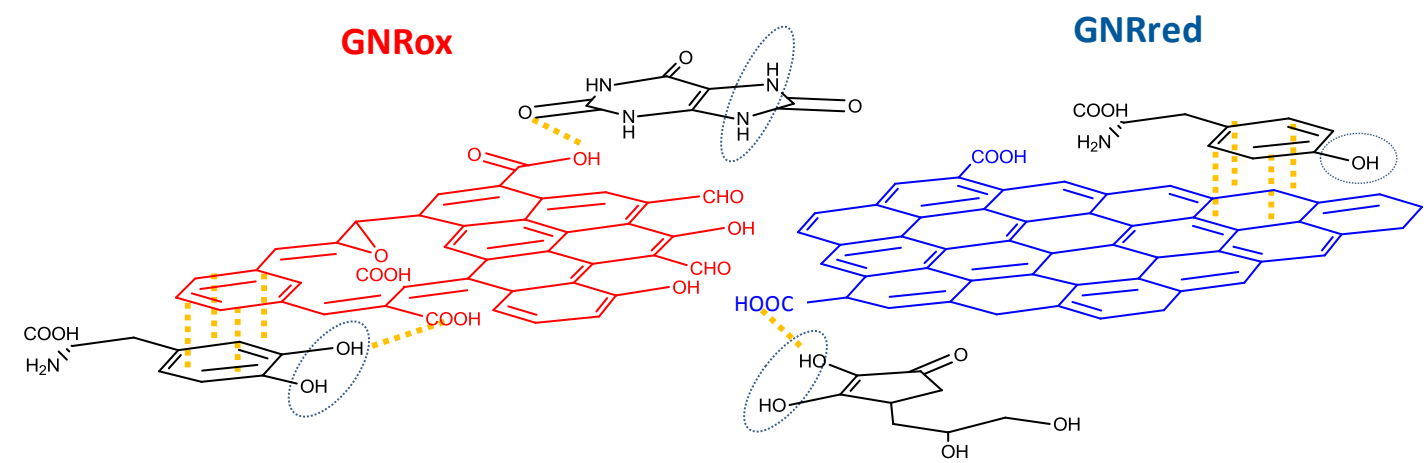

Fig. 4. (Top) Differential pulse voltammograms for $1 \mathrm{mM}$ ascorbic acid (a), LD (b), UA (c) and L-Tyr (d). (一) Bare GCE, (一) MWCNTs, (一) GNRox and (-) GNRred. (See experimental section for working conditions). (Bottom) Schematic diagram illustrating the interactions between the analytes (black) and graphene surfaces which showed the lower oxidation potential. The oxidation reaction centers are indicated by the blue circle and the main 5 interactions are indicated in yellow.

LD, which basic structure derived from CT, displayed indistinguishable behavior for GNRred and GNRox. Therefore, the predominant $\pi-\pi$ interactions plus hydro-gen interactions between the benzenediol group and the GNRred and GNRox 10 surfaces appeared to be responsible for the similar responses.

The oxidation process of L-Tyr was more difficult due to the presence of only one hydroxyl group. For this reason the similar oxidation potentials observed on all carbon materials (MWCNTs, GNRox, and GNRred) could be explained in terms of weak $\pi-\pi$ 15 interactions between the tyrosine and the carbon materials.

Fig. 5A shows the electrochemical responses of mix-tures of the three isomers: HQ, CT, and RS on GCE, MWCNTs, GNRox, and GNRred. The responses revealed that although the bare GCE did not permit the simultaneous detection of the benzene 1,2 and 20 1,4-diols, the electrocatalytic properties of GNRox and GNRred allowed the simultaneous and separate detection of the three target molecules. Fig. 5B shows that the selective detection of LD, AA, and UA could only be achieved using the GNRred electrode. Fig. 5C shows that the separate components of a 25 mixture comprising tyrosine and UA could be identified using the GNRred electrode as well. The detection of individual target molecules in a mixture relies on the availability of distinct interactions between each nanomaterial and target molecule, and consequently, to the exceptional electrocatalytic properties
30 exhibited.

The strength of the voltammetric studies was examined by measuring the technique's repeatability and repro-ducibility. The repeatability (multiple experiments con-ducted on the same day) and the reproducibility (multiple experiments conducted on 35 different days) of the oxidation peak positions was found to be excellent, with RSD values of $<4 \%(n=10)$. Good inter-electrode precision was achieved, with RSD values of $<6 \%(n=5$, same day) and $\mathrm{RSD}<9 \% \quad(\mathrm{n}=5$, different days), for the GNRred electrode. The precision was significantly better than the 40 precision obtained from the GCE electrode (RSD $<10 \% \quad n=4$ electrodes, same day).

It is worth noting that real samples were also tested. Fig. S7 illustrates the electrochemical detection of $\mathrm{HQ}$ and UA in cosmetic and urine samples, respectively. Interestingly, in both 45 cases, the GNRs displayed better intensity currents than the GCE. The excellent results in complex matrix suggest that this technique is suitable for the analysis of complex samples. Good precision was achieved for both samples. The cosmetic sample analysis was characterized by an RSD of $<0.5 \%$ for the oxidation 50 peaks and an RSD of $<2 \%$ for the peak currents. The urine sample analysis was characterized by an RSD of $<0.5 \%$ for the oxidation peaks and an RSD of $<10 \%$ for the peak currents. 

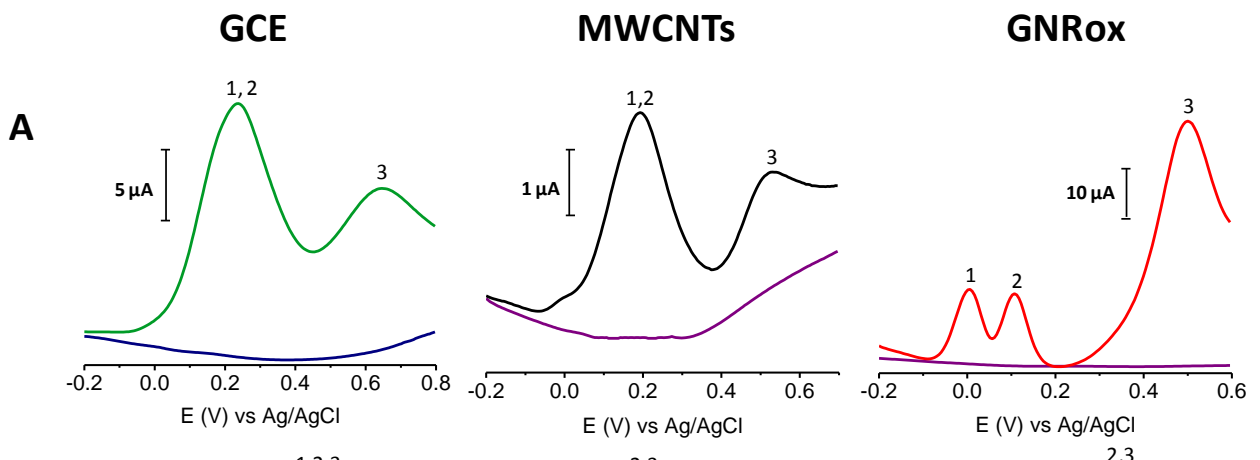

\section{GNRred}
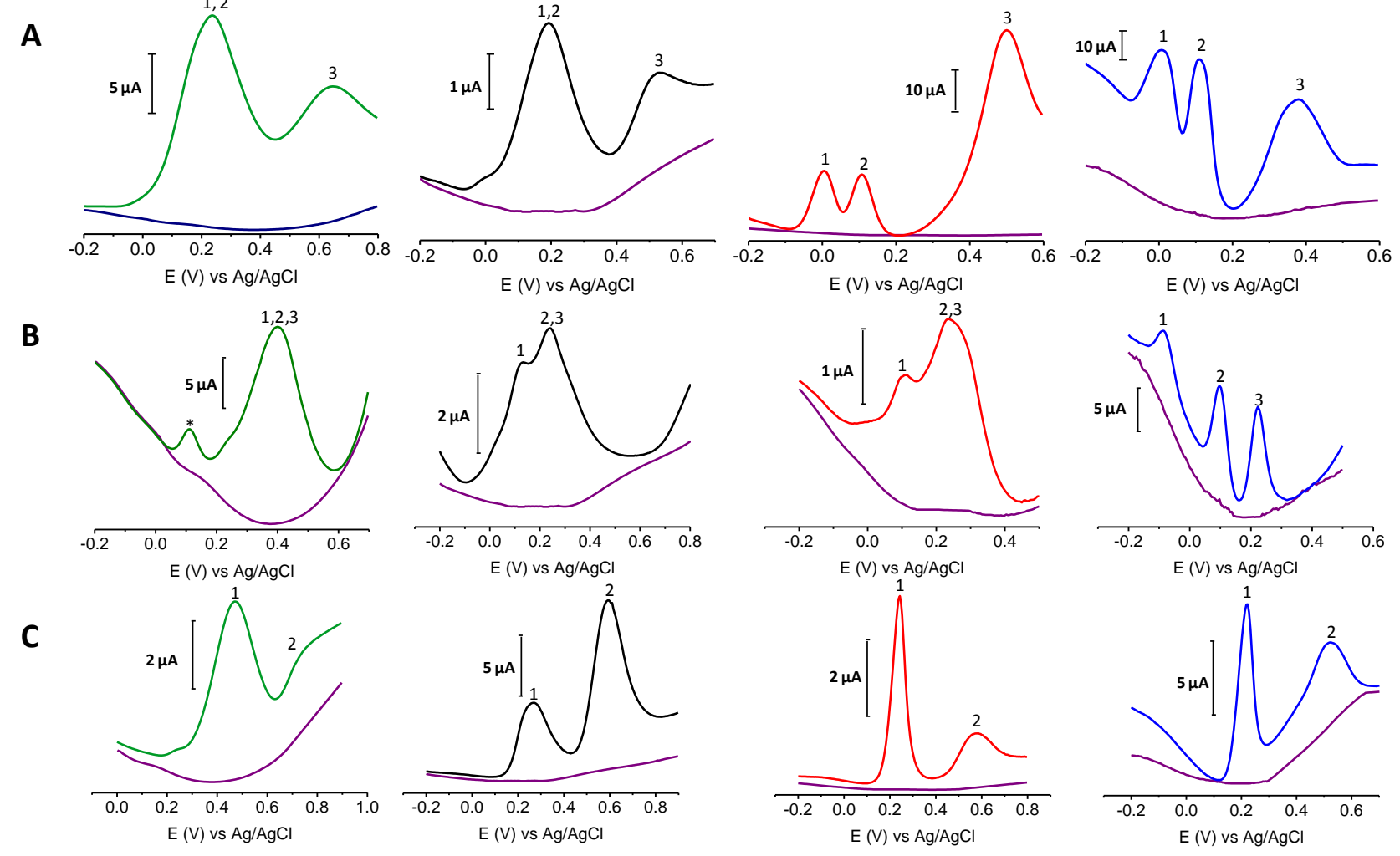

Fig. 5. (A) Voltammograms for the detection of: HQ $0.25 \mathrm{mM}$ (peak 1); CA $0.25 \mathrm{mM}$ (peak 2) and RS $1.0 \mathrm{mM}$ (peak 3). (B) AA $0.5 \mathrm{mM}$ (peak 1); LD $0.05 \mathrm{mM}$ (peak 2), and UA $0.05 \mathrm{mM}$ (peak 3). (C) UA $1.0 \mathrm{mM}$ (peak 1) and L-Tyr $1.0 \mathrm{mM}$ (peak 2). (一) Background signal, (一) Bare GCE, (一) MWCNTs, (一) GNRox, and (-) GNRred. Note: * Pre-peak oxidation in LD. (See the experimental section for a description of the working conditions.)

\section{${ }_{5}$ Conclusions}

Both graphenes displayed excellent electrochemical behaviour in the detection of the target molecules, being this behaviour rigorously attribute to graphene and to other graphitic materials. The interactions between the target molecules and the GNR 10 materials present new opportunities in the field of electrochemistry. A suitable graphene material may potentially be tailored for a par-ticular detection application with consideration for the relevant degree of oxidation and $\mathrm{sp}^{2}$ structure in the electrode that would be required to promote hydrogen bonding or ${ }_{15} \pi-\pi$ interactions between the electrode and the target molecule. GNRox appeared to be ideal for the detection of molecules having high oxidation potentials, whereas GNRred displayed a better response to aromatic molecules, such as the conjugated 1,2-diols, which inter-acted with the electrode predominantly 20 through $\pi-\pi$ interactions.

Although both graphenes exhibited excellent electro-chemical performance, GNRred became an exceptional material. The completely opened GNRred lattices, which displayed an average thickness of $1.2 \mathrm{~nm}$ and a high per-centage of $\mathrm{sp}^{2}$-carbon were 25 obtained through the syn-thetic methods described here. GNRred exhibited a 10-fold higher electrochemical surface area and a better analytical performance in the context of electrochemical sensing in comparison with the GCE. These GNRred features allowed for improved electrochemical sensing and suggested that 30 this material was suitable for the electrochemical detection of target molecules. The outstanding electrocatalytic effect performance relies on the presence of a restored $\mathrm{sp}^{2}$ structure that includes oxygen groups in the GNRred lattice. These combined features yielded excellent electrocatalytic properties due to the 35 effects of both the $\pi-\pi$ and hydrogen bonding interactions between the molecules and the GNRs. These interactions enhanced electrocatalysis at the primary catalytic sites at the oxidation centers. The studies described here demonstrate that GNRred is a promising material for use in molecular sensing, 40 with very rich chemistry and electrochemistry properties. GNRred combines the advantages derived from both the high proportion of $\mathrm{sp}^{2}$-carbon atoms available within the surface layers (similar to the structure of exfoliated graphene, $0.4 \mathrm{~nm}$ corresponds to one layer) with the advantages derived from the 45 remaining oxygen moieties present on the surface. The results presented here open new opportunities for electrochemical sensing applications and guide the process of tailoring a suitable graphene electrode material for use in a particular molecular detection application.

\section{${ }_{50}$ Acknowledgment}

Financial support provided by the Spanish Ministry of Science and Innovation (CTQ2011-28135), the Spanish ministry of Economy and Competitiveness (FIS2012-38866-C05-05 545, MICINNTEC2010-15736), as well as by the AVANSENS 55 program from the Community of Madrid (S2009/PPQ-1642) is 
gratefully acknowledged. D. Aída Martin acknowledges the FPU fellowship received from the Ministry of Education, Culture and Sports. J. Hernández-Ferrer acknowledges the Spanish Superior Council for Scientific Research (CSIC) for his JAE-Doc contract.

\section{${ }_{5}$ Notes and references}

${ }^{a}$ Department of Analytical Chemistry, Physical Chemistry and Chemical Engineer, University of Alcalá, E-28871. Alcalá de Henares, Madrid, Spain.Fax: 918854971; E-mail: alberto.escarpa@uah.es

${ }^{b}$ Instituto de Carboquímica ICB-CSIC, Miguel Luesma Castán, 4, 50018 10 Zaragoza, Spain.

${ }^{c}$ Instituto de Ciencia de Materiales de Madrid (CSIC), C/ Sor Juana Inés de la Cruz $N^{o}$ 3, 28049 Madrid, Spain.

$\dagger$ Electronic Supplementary Information (ESI) available: [details of any supplementary information available should be included here]. See 15 DOI: $10.1039 / \mathrm{b} 000000 \mathrm{x} /$

1 K. S. Novoselov, A. K. Geim, S. V. Morozov, D. Jiang, Y. Zhang, S. V. Dubonos, I. V. Grigorieva and A. A. Firsov, Science, 2004, 306, 666-669.

202 S. Park and R. S. Ruoff, Nature Nanotechnology, 2009, 4, 217-224.

3 C. Lee, X. Wei, J. W. Kysar and J. Hone, Science, 2008, 321, 385388.

4 S. Stankovich, D. A. Dikin, G. H. B. Dommett, K. M. Kohlhaas, E. J. Zimney, E. A. Stach, R. D. Piner, S. T. Nguyen and R. S. Ruoff,

25 Nature, 2006, 442, 282-286.

5 M. Terrones, Nature, 2009, 458, 845-846.

6 Liang Ma, Jinlan Wang and Feng Ding, ChemPhysChem, 2013, 14, 47-54.

7 L. Jiao, L. Zhang, X. Wang, G. Diankov and H. Dai, Nature, 2009, $30 \quad \mathbf{4 5 8}, 877-880$.

8 D. V. Kosynkin, A. L. Higginbotham, A. Sinitskii, J. R. Lomeda, A. Dimiev, B. K. Price and J. M. Tour, Nature, 2009, 458, 872-U5.

9 C. K. Chua, Z. Sofer and M. Pumera, Chemistry-an Asian Journal, 2012, 7, 2367-2372.

3510 S. Vadahanambi, J. Jung, R. Kumar, H. Kim and I. Oh, Carbon, 2013, 53, 391-398.

11 A. G. Cano-Marquez, F. J. Rodriguez-Macias, J. Campos-Delgado, C. G. Espinosa-Gonzalez, F. Tristan-Lopez, D. Ramirez-Gonzalez, D. A. Cullen, D. J. Smith, M. Terrones and Y. I. Vega-Cantu, Nano

40 Letters, 2009, 9, 1527-1533.

12 L. Ci, Z. Xu, L. Wang, W. Gao, F. Ding, K. F. Kelly, B. I. Yakobson and P. M. Ajayan, Nano Research, 2008, 1, 116-122.

13 K. Kim, A. Sussman and A. Zettl, Acs Nano, 2010, 4, 1362-1366.

14 X. Yang, X. Dou, A. Rouhanipour, L. Zhi, H. J. Raeder and K. Muellen, J. Am. Chem. Soc., 2008, 130, 4216-4217.

15 M. Terrones, A. R. Botello-Mendez, J. Campos-Delgado, F. LopezUrias, Y. I. Vega-Cantu, F. J. Rodriguez-Macias, A. L. Elias, E. Munoz-Sandoval, A. G. Cano-Marquez, J. Charlier and H. Terrones, Nano Today, 2010, 5, 351-372.

5016 Y. Guo, W. Guo and C. Chen, Appl. Phys. Lett., 2008, 92, 243101.

17 D. B. Shinde, J. Debgupta, A. Kushwaha, M. Aslam and V. K. Pillai, J. Am. Chem. Soc., 2011, 133, 4168-4171.

18 M. Pumera, A. Ambrosi, A. Bonanni, E. L. K. Chng and H. L. Poh, Trac-Trends in Analytical Chemistry, 2010, 29, 954-965.

5519 D. A. C. Brownson and C. E. Banks, Analyst, 2010, 135, 2768-2778.

20 Y. Shao, J. Wang, H. Wu, J. Liu, I. A. Aksay and Y. Lin, Electroanalysis, 2010, 22, 1027-1036.

21 S. Wu, Q. He, C. Tan, Y. Wang and H. Zhang, Small, 2013, 9, 11601172 .

6022 S. Zhang, S. Tang, J. Lei, H. Dong and H. Ju, J Electroanal Chem, 2011, 656, 285-288.

23 F. Valentini, D. Romanazzo, M. Carbone and G. Palleschi, Electroanalysis, 2012, 24, 872-881.

24 X. Dong, Q. Long, J. Wang, M. B. Chan-Park, Y. Huang, W. Huang 65 and P. Chen, Nanoscale, 2011, 3, 5156-5160.

25 F. Valentini, M. Carbone and G. Palleschi, Analytical and Bioanalytical Chemistry, 2013, 405, 3449-3474.
26 Y. Yang, J. Zhou, H. Zhang, P. Gai, X. Zhang and J. Chen, Talanta, 2013, 106, 206-211.

7027 R. K. Srivastava, S. Srivastava, T. N. Narayanan, B. D. Mahlotra, R. Vajtai, P. M. Ajayan and A. Srivastava, Acs Nano, 2012, 6, 168-175.

28 X. Shen, Y. Cui, Y. Pang and H. Qian, Electrochim. Acta, 2012, 59, 91-99.

29 S. Wu, X. Lan, F. Huang, Z. Luo, H. Ju, C. Meng and C. Duan, 75 Biosens. Bioelectron., 2012, 32, 293-296.

30 J. Tang, L. Hou, D. Tang, J. Zhou, Z. Wang, J. Li and G. Chen, Biosens. Bioelectron., 2012, 38, 86-93.

31 M. S. Goh and M. Pumera, Analytical and Bioanalytical Chemistry, 2011, 399, 127-131.

8032 S. M. Tan, C. K. Chua and M. Pumera, Analyst, 2013, 138, 17001704.

33 C. Sun, C. Chang, H. Lee, J. Zhou, J. Wang, T. Sham and W. Pong, Acs Nano, 2011, 5, 7788-7795.

34 S. Suresh, V. Srivastava and I. M. Mishra, IJEEE, 2012, 3, 32.

8535 J. M. Zen, C. T. Hsu, Y. L. Hsu, J. W. Sue and E. D. Conte, Anal. Chem., 2004, 76, 4251-4255.

36 A. Salimi, H. MamKhezri and R. Hallaj, Talanta, 2006, 70, 823-832.

37 K. Reddaiah, T. M. Reddy and P. Raghu, J Electroanal Chem, 2012, 682, 164-171.

9038 R. D. Oneill, Analyst, 1994, 119, 767-779.

39 Z. Wang, H. Hu, Y. Wei and Q. Huang, Physica B-Condensed Matter, 2010, 405, 3895-3898.

40 M. Roos, D. Kuenzel, B. Uhl, H. Huang, O. B. Alves, H. E. Hoster, A. Gross and R. J. Behm, J. Am. Chem. Soc., 2011, 133, 9208-9211.

9541 M. V. Antisari, R. Marazzi and R. Krsmanovic, Carbon, 2003, 41, 2393-2401.

42 A. M. Benito, W. K. Maser and M. T. Martinez, International Journal of Nanotechnology, 2005, 2, 71-89.

43 X. Gao, J. Jang and S. Nagase, Journal of Physical Chemistry C, $100 \quad 2010,114,832-842$.

44 F. C. Anson, Anal. Chem., 1964, 36, 932-934.

45 F. C. Anson and R. A. Osteryoung, J. Chem. Educ., 1983, 60, 293296.

46 N. P. C. Stevens, M. B. Rooney, A. M. Bond and S. W. Feldberg,

105 Journal of Physical Chemistry a, 2001, 105, 9085-9093.

47 M. Zhou, Y. Zhai and S. Dong, Anal. Chem., 2009, 81, 5603-5613.

48 A. Ambrosi, A. Bonanni, Z. Sofer, J. S. Cross and M. Pumera, Chemistry-a European Journal, 2011, 17, 10763-10770.

49 E. B. Barros, K. Sato, G. G. Samsonidze, A. G. Souza Filho, M. S. 110 Dresselhaus and R. Saito, Physical Review B, 2011, 83, 245435.

50 S. Stankovich, D. A. Dikin, R. D. Piner, K. A. Kohlhaas, A. Kleinhammes, Y. Jia, Y. Wu, S. T. Nguyen and R. S. Ruoff, Carbon, 2007, 45, 1558-1565.

51 M. Cheng, R. Yang, L. Zhang, Z. Shi, W. Yang, D. Wang, G. Xie, D. 115 Shi and G. Zhang, Carbon, 2012, 50, 2581-2587.

52 A. Lerf, H. Y. He, M. Forster and J. Klinowski, J Phys Chem B, 1998, 102, 4477-4482.

53 H. Y. He, J. Klinowski, M. Forster and A. Lerf, Chemical Physics Letters, 1998, 287, 53-56. 